\title{
Influence of Diabetes Mellitus on the Biomechanical Properties of the Human Skin as Measured Using the Cutometer: A Case-Control Study
}

\section{Ji Woong Park}

Department of Physical Medicine and Rehabilitation, Soonchunhyang University Seoul Hospital, Soonchunhyang University College of Medicine, Seoul, Korea

\begin{abstract}
Objective: Longstanding diabetes mellitus has been suggested to cause several skin problems associated with biomechanical skin properties. However, the association between impaired skin properties and diabetic peripheral neuropathy (DPN) has not been clarified. This study aimed to investigate the biomechanical properties of the diabetic human skin and their relationship with DPNs. Methods: This case-control study included 39 chronic diabetic patients and 41 matched healthy controls. The diabetic group was divided into subgroups according to the presence of diabetic sensorimotor polyneuropathy (DSPN) and peripheral autonomic neuropathy (PAN) as determined by the results of nerve conduction study and sympathetic skin response test, respectively. Different skin regions were measured using the non-invasive Cutometer instrument (Courage and Khazaka Electronic GmbH, Cologne, Germany). The following skin parameters were compared between the groups: distensibility (Uf), elasticity (Ua/Uf and Ur/Uf), and viscoelasticity (Uv/Ue). The impact of DPNs, participant age, and diabetes duration on the investigated skin properties was analyzed.

Results: The diabetic group showed higher Uf values, and lower Ua/Uf, Ur/Uf, and Uv/Ue values, compared to the control group. However, no significant differences in biomechanical skin properties were noted between diabetic patients with PAN and without PAN. There were also no differences in the investigated skin properties between diabetic patients with DSPN and without DSPN. Diabetes duration and participant age of both groups had a significant impact on the skin properties.

Conclusion: A long-term hyperglycemic state could induce inferior biomechanical skin properties in the peripheral limbs; however, this phenomenon seems unrelated to DPNs.
\end{abstract}

Keywords: Skin; Diabetes mellitus; Elasticity; Cutometer; Peripheral autonomic neuropathy

\section{INTRODUCTION}

Longstanding diabetes causes several skin changes and complications. However, the complications might be unnoticed because they do not result in serious sequelae, except for pressure ulcers and the diabetic foot syndrome. Diabetes mellitus (DM) is multisystemic, with effects on the metabolic, immune, vascular, and nervous systems. Altered physiologic conditions following diabetes might directly impact cutaneous functions. Longstanding hyperglycemia can give rise to diabetic polyneuropathies (DPNs) which can be classified into typical and atypical subgroups [1]. Typical DPN is commonly known as diabetic sensorimotor polyneuropathy (DSPN), and among atypical DPNs, peripheral auto- nomic neuropathy (PAN) can cause several pathological skin conditions, including dryness, fissure, and ulceration due to abnormal sympathetic function [2-4]. Peripheral sympathetic function can be evaluated with the sympathetic skin response (SSR) test, which is a simple non-invasive test for assessing small-fiber sudomotor function $[5,6]$. Bioengineering methods have been employed as quantitative tools in the study of both normal skin and skin diseases which affect skin mechanics. The Cutometer (Courage and Khazaka Electronic GmbH, Cologne, Germany) is a non-invasive in vivo suction device, which can evaluate the biomechanical skin properties of distensibility, elasticity, and viscoelasticity. All the parameters measured by the Cutometer (Courage and Khazaka Electronic $\mathrm{GmbH}$ ) are related to skin composition and structure, 
and measurements show high inter-rater and intra-rater reliability and validity [7]. Different skin layer depths can be examined, depending on the aperture size. Consequently, a large 8-mm diameter measuring probe determines the properties of the entire skin layer, while a small 2-mm probe assesses only the epidermis and papillary dermis [8]. In a previous study using the small 2-mm aperture probe, altered biomechanical properties in the epidermis were noted in diabetics [9]. However, prolonged hyperglycemia could impair collagen homeostasis leading to diabetic dermal thickness [10]. Moreover, following spinal cord injury, sympathetic denervated dermatomes were markedly involved in the pathologic process in skin measured by the Cutometer (Courage and Khazaka Electronic $\mathrm{GmbH}$ ) with a large 8-mm probe [11]. However, no study has evaluated the biomechanical properties of the entire skin layer of diabetic patients with respect to the presence of DPNs.

Therefore, this study attempted to (1) assess the biomechanical properties of all layers of the diabetic skin, and (2) determine the relationship of these biomechanical properties with the presence of PAN or DSPN.

\section{MATERIALS AND METHODS}

\section{Study design}

This study used a case-control design and was performed at the Soonchunhyang University Hospital (SCHUH) in Seoul, the Republic of Korea from March 2010 to February 2011. The study was approved by the Institutional Review Board of SCHUH (IRB No., SCHUH 2009-011). Before inclusion in the study, each participant provided written informed consent. Thirty-nine patients with chronic diabetes and 41 sex- and age-matched healthy controls were recruited (Fig. 1). Because sex and age can affect the biomechanical characteristics of the human skin, sex and age at 10-year intervals were chosen at a 1:1 ratio to match the participant's characteristics. Nerve conduction studies (NCS) and SSR tests for subgroup analysis based on the presence of DSPN and PAN were performed for the diabetic group.

\section{Participants}

The diabetic group included participants aged 19 years and above who had diabetes for more than one year prior to the time of the study. We noted their types of diabetes, fasting plasma glucose (FPG) and hemoglobin Alc (HbAlc), smoking history, and anthropometric parameters including weight, height, and body mass

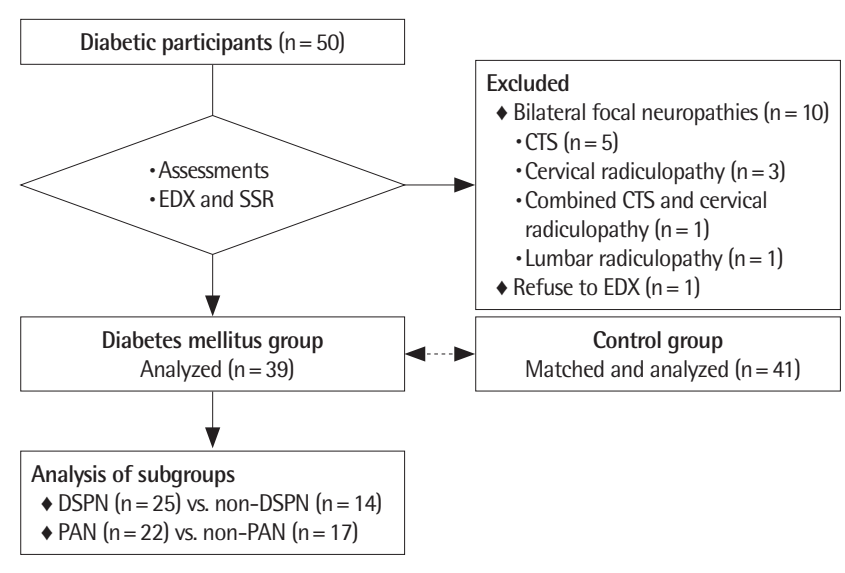

Fig. 1. A flow chart of the study design. EDX, electrodiagnostic study; SSR, sympathetic skin response; CTS, carpal tunnel syndrome; DSPN, diabetic sensorimotor polyneuropathy; PAN, peripheral autonomic neuropathy.

index (BMI). We also collected the same information in the control group. We excluded subjects with: (1) skin lesions at the measurement site (e.g., burn, scar, keloid, etc.); (2) history of skin disorders or systemic disorders with the potential to influence biomechanical skin properties; (3) focal neuropathies of extremities (e.g., radiculopathy and carpal tunnel syndrome); (4) central nervous system lesions; and/or (5) the inability to assume a proper posture because of musculoskeletal deformities.

\section{Electrodiagnostic study}

A physiatrist with more than 10-years experience in electrodiagnosis performed the electrodiagnostic study including SSR using a Medelec Synergy Mobile 5-channel device (Oxford Instruments, Abingdon, UK). NCS was performed for both upper and lower limbs to identify DSPN and focal entrapment neuropathies or radiculopathies. Based on a previous guideline [12], the diagnostic criteria for DSPN included a (1) sural sensory action potential of $<7.3 \mu \mathrm{V}$, and (2) peroneal motor conduction velocity of $<43.9 \mathrm{~m} / \mathrm{sec}$ recorded at the same time during NCS. When the patients were suspected with focal entrapment neuropathies, as determined by the observation of known symptoms and via NCS results, we performed additional needle electromyography to determine the diagnosis. SSR test results indicate the changes in skin electrical potential and reflect sudomotor function. The SSR method used in this study was similar to that previously described [13]: the active electrodes were attached to both palms and soles, which are areas with the highest density of eccrine sweat glands in the body. Reference electrodes were also placed on the dorsum of 
the hands and feet. The median and tibial nerves were then stimulated with an intensity of $20 \mathrm{~mA}$ and a pulse duration of $0.2 \mathrm{~ms}$, and the potentials were recorded via the ipsilateral and contralateral electrodes using a 4-channel recording system. When the potentials generated after the electrical stimulations were simultaneously recorded at the four extremities, it was considered an "abnormal SSR" test, representing a situation in which there were no responses in at least two limbs [13].

\section{Skin measurements}

All outcomes were measured at one visit. Subjects were placed in the supine position and allowed to acclimatize to room conditions. The air flow and room temperature were maintained at $20^{\circ} \mathrm{C}$ to $25^{\circ} \mathrm{C}$ by an air conditioner, while the relative humidity was kept at $40 \%$ to $50 \%$ [14]. Biomechanical skin properties were determined with the non-invasive suction device, the Cutometer MPA 580 probe (Courage and Khazaka Electronic GmbH). Using an optical unit, the Cutometer (Courage and Khazaka Electronic
$\mathrm{GmbH}$ ) evaluates vertical skin deformation using a controlled vacuum produced by $8 \mathrm{~mm}$-diameter probes. The time/strain mode was selected, with five consecutive cycles comprising a 5 -second application of vacuum at $450 \mathrm{mbar}$, followed by a 3-second relaxation period (Fig. 2) [15]. We measured the following biomechanical skin properties based on previous guidelines [8]: distensibility (Uf, final distension), elasticity (Ua/Uf, gross elasticity; Ur/Uf, biologic elasticity), and viscoelasticity (Uv/Ue, viscoelasticity). The details of the body regions measured are shown in Fig. 2.

\section{Statistical analysis}

To estimate the sample size, we referred to a previous study that compared elasticity (Ua/Uf) values based on the presence of sympathetic paralysis at the measured region in patients with spinal cord injury [11]. To detect this difference in a case-control study using the Student t-test, assuming a two-tailed alpha level of 0.01, and $90 \%$ power, the suggested sample size was 38 subjects per group. The normality of distribution was evaluated with the Shap-
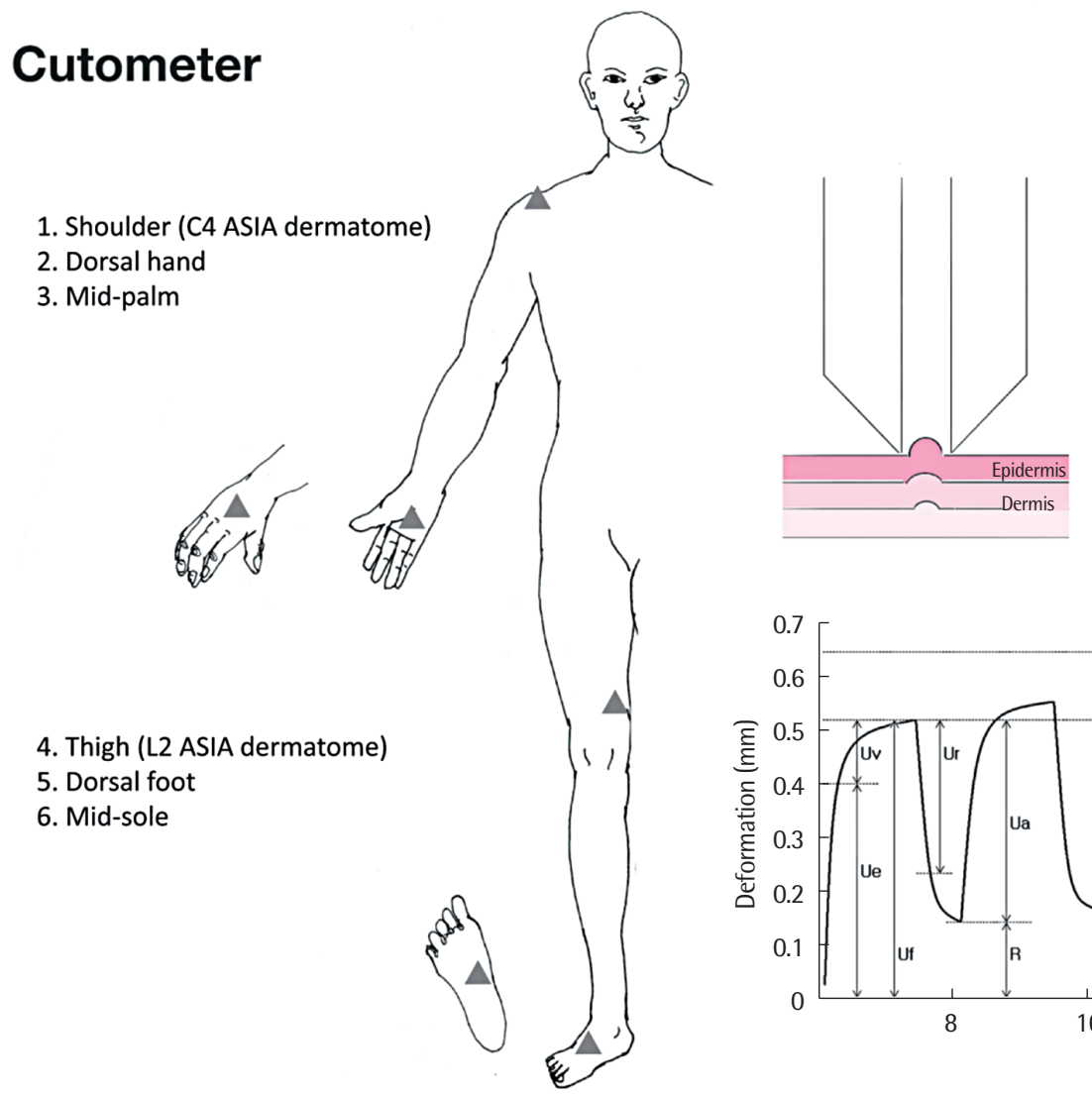

Uf: final distension

Ue: immediate distension

Ur: immediate retraction

Uv: delayed distension

Ua/Uf: gross skin elasticity

Ur/Uf: biologic skin elasticity

Uv/Ue: skin viscoelasticity

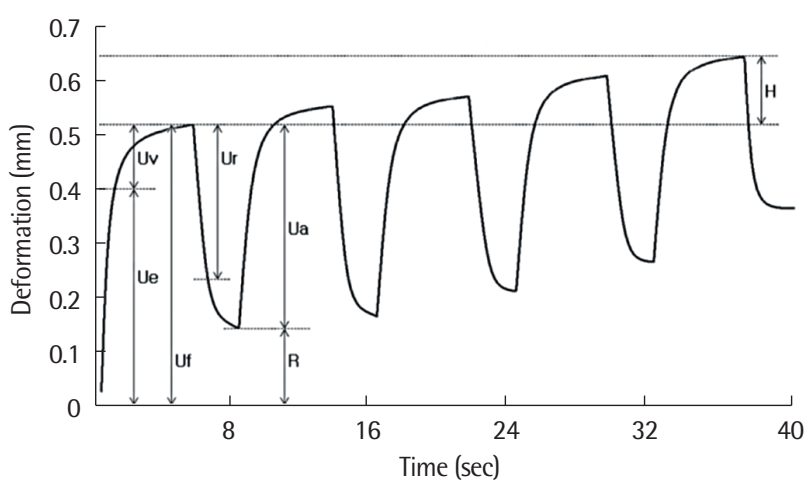

Fig. 2. Skin deformation curve (right lower) was obtained using the Cutometer MPA 580 instrument (Courage and Khazaka Electronic GmbH, Cologne, Germany) for skin measurements at different body regions (triangles). Absolute and relative parameters (right upper) were obtained from the illustrated curve. ASIA, American Spinal Injury Association. 
iro-Wilk test. All demographic data are presented as mean and standard deviation (SD). The Student t-test was used to compare most continuous variables and the chi-square test was used to compare the categorical variables, such as sex and smoking ratio, between both groups. Pearson's correlation was used to evaluate the association of the skin variables with participant age and diabetes duration. Variables with $\mathrm{P}<0.05$ were considered statistically significant. The SPSS ver. 14.0 software for Windows (SPSS Inc., Chicago, IL, USA) was used to perform the data analyses.

\section{RESULTS}

Fifty diabetic participants were initially screened (Fig. 1). Of these, 10 were excluded and one refused the electrodiagnostic study. Therefore, 39 participants were included in the diabetic group. Thereafter, 41 age- and sex-matched controls were recruited. The 39 diabetic participants were divided into subgroups based on the electrodiagnostic studies and SSR tests. The participant characteristics are summarized in Table 1. No significant differences with respect to age, sex, smoking, weight, height, and BMI were noted between both groups. In the diabetic and control groups, the mean \pm SD age was $59.9 \pm 8.7$ years and $59.4 \pm 9.7$

Table 1. Baseline demographics of participants

\begin{tabular}{|c|c|c|c|}
\hline \multirow[b]{2}{*}{ Variable } & \multicolumn{2}{|c|}{ Group } & \multirow[b]{2}{*}{ P-value } \\
\hline & $\begin{array}{l}\text { Diabetes } \\
\text { mellitus } \\
(\mathrm{N}=39)\end{array}$ & $\begin{array}{l}\text { Control } \\
(\mathrm{N}=41)\end{array}$ & \\
\hline Age (yr) & $59.9 \pm 8.7$ & $59.4 \pm 9.7$ & $0.82^{\mathrm{a})}$ \\
\hline Sex (female) & $21(53.8)$ & $21(51.2)$ & $0.83^{b)}$ \\
\hline Height (cm) & $162.7 \pm 7.1$ & $161.1 \pm 6.6$ & $0.30^{\mathrm{al}}$ \\
\hline Weight (kg) & $63.7 \pm 8.5$ & $61.4 \pm 7.7$ & $0.22^{\mathrm{al}}$ \\
\hline Body mass index $\left(\mathrm{kg} / \mathrm{m}^{2}\right)$ & $24.2 \pm 3.9$ & $23.7 \pm 2.5$ & $0.48^{\mathrm{a})}$ \\
\hline Smoker & $6(15.4)$ & $6(14.6)$ & $0.93^{b)}$ \\
\hline Diabetes duration (yr) & $15.6 \pm 8.9$ & NA & \\
\hline Fasting plasma glucose (mg/dL) & $145.5 \pm 33.2$ & NA & \\
\hline Hemoglobin A1c (\%) & $6.2 \pm 1.3$ & NA & \\
\hline \multicolumn{4}{|l|}{ Types of diabetes } \\
\hline Type 1 & $7(17.9)$ & NA & \\
\hline Type 2 & $32(82.1)$ & NA & \\
\hline \multicolumn{4}{|l|}{ Incidence of diabetic polyneuropathies } \\
\hline Diabetic sensorimotor polyneuropathy & $25(64.1)$ & NA & \\
\hline Peripheral autonomic neuropathy & $22(56.4)$ & NA & \\
\hline
\end{tabular}

Values are presented as mean \pm standard deviation or number $(\%)$. NA, not applicable.

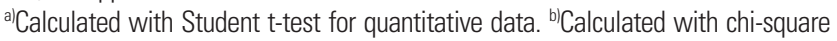
test for qualitative data. years, respectively. In the diabetic group, the mean diabetes duration was $15.6 \pm 8.9$ years. Of the 39 diabetic participants, 7 (17.9\%) had type 1 diabetes, and 32 (82.1\%) had type 2 diabetes. In the diabetic group, DSPN and PAN were observed in 25 cases (64.1\%) and 22 cases (56.4\%), respectively. FPG and HbAlc were $145.5 \pm$ $33.2 \mathrm{mg} / \mathrm{dL}$ and $6.2 \% \pm 1.3 \%$, respectively. Additionally, there were no differences in the values between the two subgroups of DSPN and PAN.

To investigate the influence of long-term diabetes on the biomechanical skin properties, we compared the mean values between the two groups. In all the measured regions, the diabetic group $(n=39)$ showed significantly different outcomes compared to the control group ( $\mathrm{n}=41)$ : increased distensibility (Uf, $\mathrm{P}<0.001)$, decreased elasticity (Ua/Uf, $\mathrm{P}<0.01$; Ur/Uf, $\mathrm{P}<0.001)$ and decreased viscoelasticity (Uv/Ue, $\mathrm{P}<0.001)$ (Fig. 3). However, no significant differences in biomechanical skin properties were noted between diabetic patients with PAN $(n=22)$ and without PAN $(n=17)$ (Fig. 4). There were also no differences in the biomechanical skin properties between patients with DSPN $(n=25)$ and without DSPN $(n=14)$. The Pearson's correlation coefficients between skin variables and participant age, as well as the duration of diabetes, were analyzed. In the control group, when biomechanical skin properties were measured in the dorsal aspects of both the hand and foot, age showed a positive correlation with Uf, and negative correlations with Ua/Uf, Ur/Uf, and Uv/Ue. The diabetic group also showed similar patterns compared to those of the control group. Compared to age, disease duration showed a relatively weak relationship with the biomechanical skin properties. Diabetic duration showed a positive correlation with Uf $(\mathrm{r}=0.285, \mathrm{P}=0.04$ in the hand dorsum; $\mathrm{r}=0.334, \mathrm{P}=0.019$ in the foot dorsum), and a negative correlation with $\mathrm{Ur} / \mathrm{Uf}(\mathrm{r}=-0.379, \mathrm{P}=0.009$ in the foot dorsum) (Table 2).

\section{DISCUSSION}

This study's findings confirmed that the presence of prolonged hyperglycemia could lead to inferior biomechanical skin properties on the peripheral limbs compared to the non-diabetic skin; however, this phenomenon seems unrelated to DPNs. As a noninvasive bioengineering tool, the Cutometer (Courage and Khazaka Electronic $\mathrm{GmbH}$ ) is beneficial for the quantitative assessment of the biomechanical properties of the diabetic skin. Common skin disorders associated with diabetes include pigment disorders, 

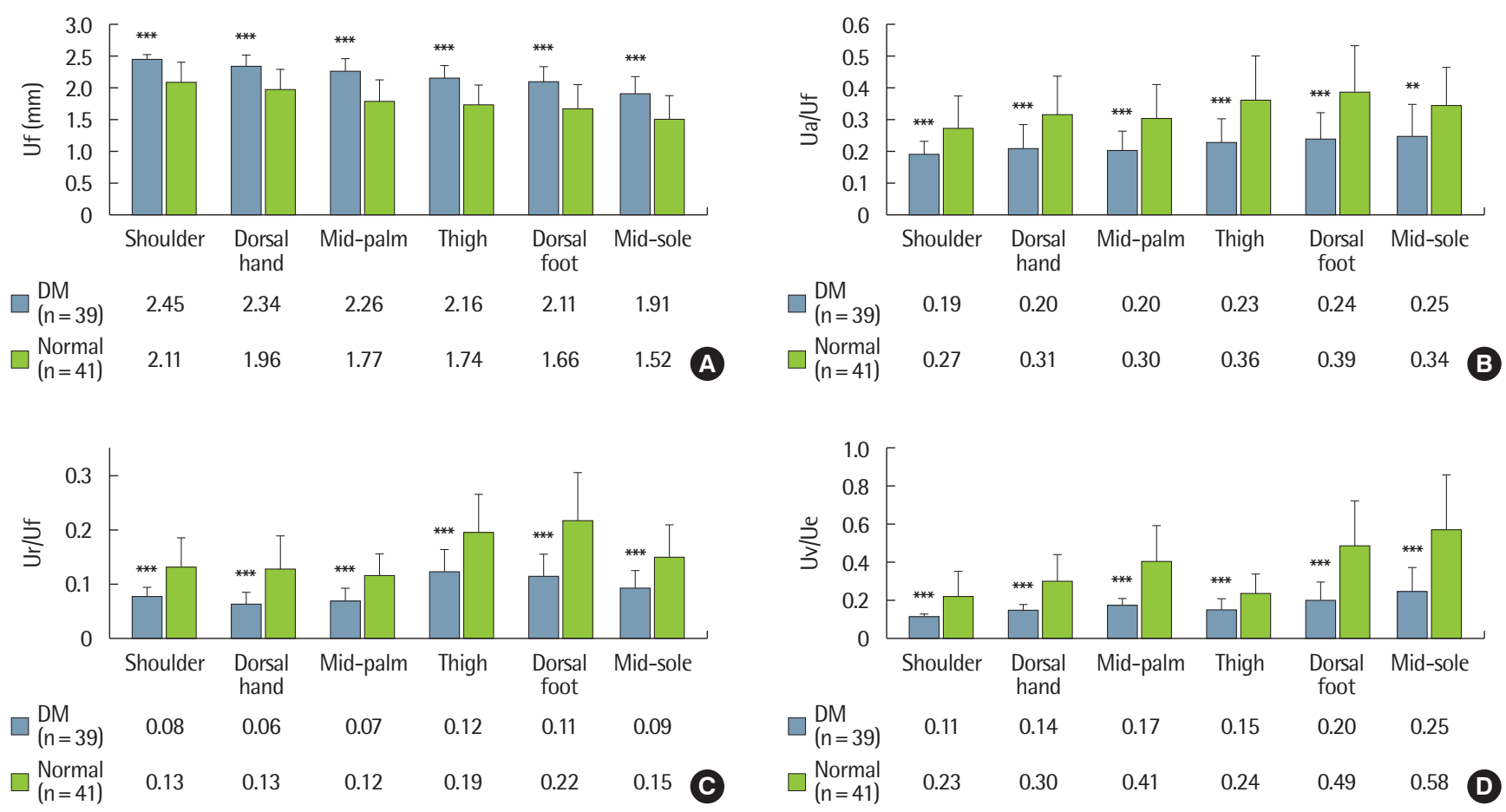

Fig. 3. Comparisons of biomechanical skin properties between the DM and control groups. The diabetic group showed increased distensibility (A), decreased elasticity $(\mathrm{B}$ and $\mathrm{C})$, and viscoelasticity (D) in all the skin regions measured. The values are presented as the mean and standard deviation bar. A Student t-test was used to compare the means of the DM and control groups. DM, diabetes mellitus; Uf, final distension; Ua/Uf, gross elasticity; Ur/Uf, biologic elasticity; Uv/Ue, viscoelasticity. ${ }^{* *} \mathrm{P}<0.01$. ${ }^{* * *} \mathrm{P}<0.001$.
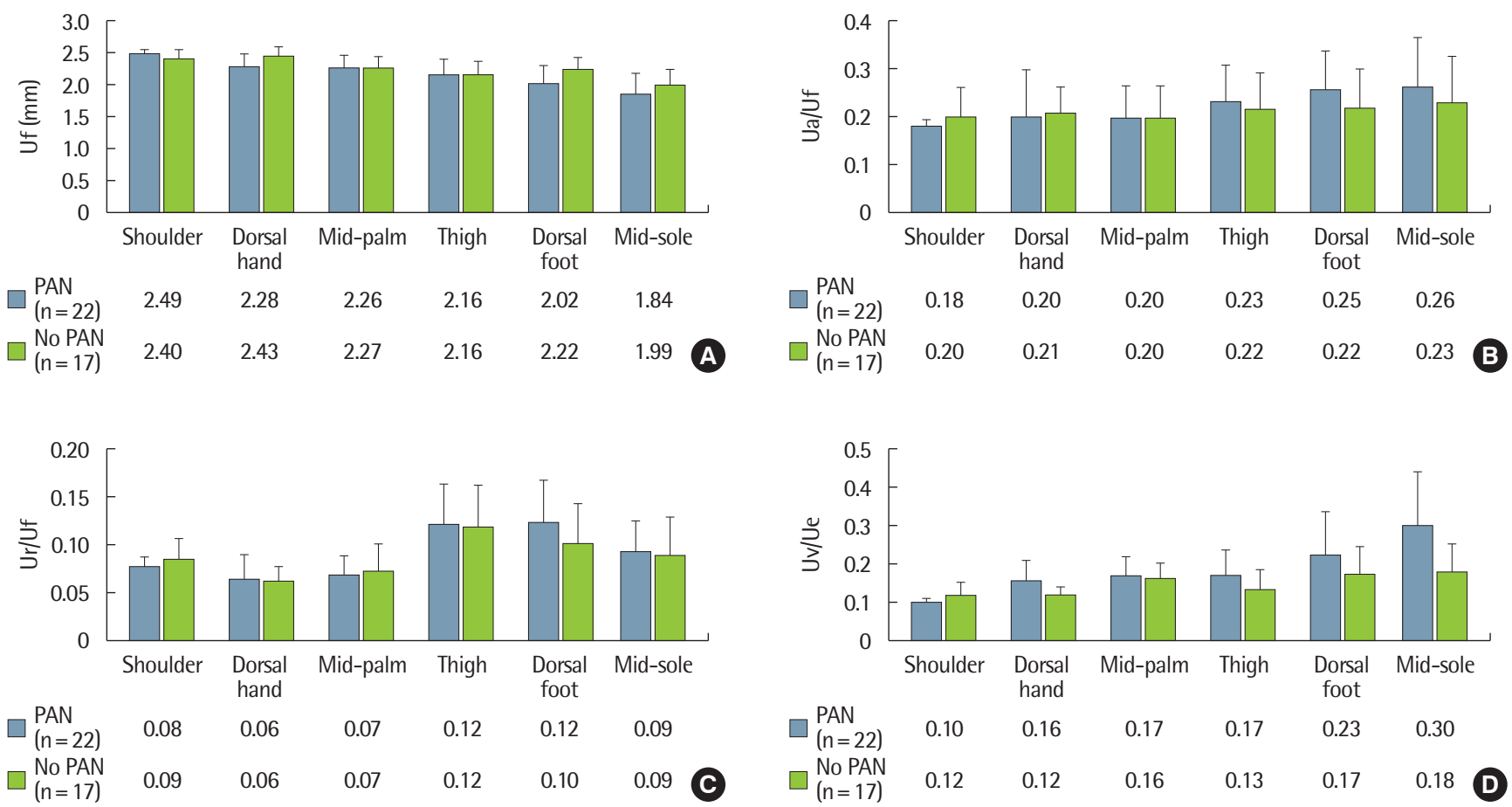

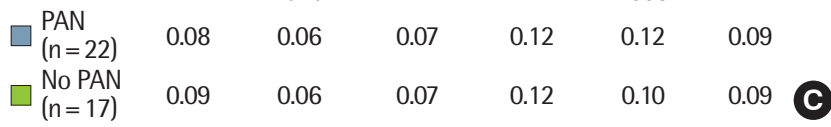

Fig. 4. (A-D) Comparisons of biomechanical skin properties between diabetic patients with abnormal and normal SSR test results. There were no differences in skin variabes between the two groups. The values are presented as the mean and standard deviation bar. A Student t-test was used to compare the means of the two groups. PAN, peripheral autonomic neuropathy; SSR, sympathetic skin response; Uf, final distension; Ua/Uf, gross elasticity; Ur/Uf, biologic elasticity; Uv/Ue, viscoelasticity. 
Table 2. Correlations of participants' age and diabetes duration with biomechanical skin variables

\begin{tabular}{|c|c|c|c|}
\hline \multirow{3}{*}{ Variable } & \multicolumn{3}{|c|}{ Correlation coefficient (r) } \\
\hline & \multicolumn{2}{|c|}{ Diabetes mellitus ( $\mathrm{N}=39$ ) } & \multirow{2}{*}{$\begin{array}{c}\text { Control }(\mathrm{N}=41) \\
\text { Age }\end{array}$} \\
\hline & Age & Diabetes duration & \\
\hline \multicolumn{4}{|c|}{ Dorsal hand } \\
\hline Uf & $0.497^{* *}$ & $0.285^{*}$ & $0.609^{* *}$ \\
\hline $\mathrm{Ua} / \mathrm{Uf}$ & $-0.468^{* *}$ & -0.107 & $-0.586^{* *}$ \\
\hline Ur/Uf & $-0.441^{* *}$ & -0.133 & $-0.562^{* *}$ \\
\hline Uv/Ue & $-0.520^{* *}$ & -0.182 & $-0.599 * *$ \\
\hline \multicolumn{4}{|c|}{ Dorsal foot } \\
\hline Uf & $0.318^{*}$ & $0.334^{*}$ & $0.777^{* *}$ \\
\hline $\mathrm{Ua} / \mathrm{Uf}$ & $-0.306^{*}$ & -0.252 & $-0.725^{* *}$ \\
\hline Ur/Uf & $-0.300^{*}$ & $-0.379^{* *}$ & $-0.674^{* *}$ \\
\hline Uv/Ue & $-0.335^{*}$ & -0.090 & $-0.733^{* *}$ \\
\hline
\end{tabular}

Values are presented as coefficient (r) by Pearson's correlation test. Uf, final distension; Ua/Uf, gross elasticity; Ur/Uf, biologic elasticity; Uv/Ue, viscoelasticity.

${ }^{*} \mathrm{P}<0.05 .{ }^{*} \mathrm{P}<0.01$.

sclerosis, erythema, pruritus, cutaneous deposition disorders, and various skin infections $[16,17]$. Combined diabetes-associated metabolic, immunologic, vascular, and nervous dysfunctions may contribute to the skin problems. Combined angiopathy and polyneuropathy is known to induce diabetic foot syndrome and pressure ulcer, through the disruption of vascular supply and neural control, as well as possible changes in biomechanical skin properties [16]. The change in diabetic skin properties might be induced by systemic influences through biochemical and pathological reactions that can lead to an altered composition of the extracellular matrix and connective tissue and functional deficits of proteins $[18,19]$. In a previous study, the tensile properties of the biopsied skin of DM patients were analyzed, using a multiaxial tensile testing device [20]. Increased stiffness of the skin was observed and the authors suggested that the changes were probably due to increased cross-linking of collagen molecules [20]. It has been shown that non-enzymatic glycosylation may play an important role in altering structural proteins such as dermal collagen [21]. Similarly, scleroderma-like skin changes are a common cutaneous manifestation in diabetic patients [22]. The main alteration in the sclerodermatous skin is increased skin thickness localized in the reticular dermis and subcutis; therefore, the large measuring probe provides more relevant information [23]. To evaluate the biomechanical alterations of the whole skin layer of diabetic skin, we used the large $8-\mathrm{mm}$ diameter Cutometer (Courage and Khazaka Electronic $\mathrm{GmbH}$ ) probe. In this study, decreased elas- ticity (Ua/Uf and Ur/Uf) was observed in the diabetic skin. The thickening of the diabetic skin can explain why the elasticity of the whole skin layer was markedly reduced. Moreover, the dermal collagen bundles might cross-link with each other, which may increase the friction between collagen fibers, and inhibit the movement of interstitial fluid in the reticular dermis. This can also explain the decreased viscoelasticity (Uv/Ue) and decreased elasticity. Furthermore, markedly increased dermal distension can cause a relative decrease in the ratio of viscoelastic to elastic distension, because $\mathrm{Uv} / \mathrm{Ue}$ is a relative parameter of viscoelasticity.

Another possible cause of skin changes may be caused by PAN, which causes peripheral sympathetic dysfunction that can control sudomotor, pilomotor, and vasomotor functions. In this study, we used the SSR test to detect PAN because it has been reported that PAN symptoms are significantly associated with abnormal SSR findings in DPN patients' limbs [5]. However, we could not demonstrate any relationship between PAN and the biomechanical properties of the diabetic skin, which was definitely observed in patients with chronic spinal cord injury: decreased distensibility, increased elasticity, and increased viscoelasticity [11]. These findings suggest that the metabolic process in diabetes may be strong enough to mask the impact of sympathetic dysfunction on biomechanical skin properties. In line with results from previous studies $[14,24]$, we found that participant age and diabetes duration were well correlated with most of the biomechanical skin parameters in the diabetic and control groups. This study had some limitations. We relied on the SSR results without considering additional clinical symptoms when we assigned the DPN subgroups. Although abnormal SSR results have been reported to be associated with major PAN symptoms, it may be difficult to generalize the diagnosis of PAN depending on SSR test results alone. Moreover, there was no attempt to quantify water intake and drug use which could affect skin barrier function. Further studies could aim to identify the alteration of cutaneous biomechanics in the diabetic skin through molecular biologic work.

In conclusion, non-invasive bioengineering methods are useful for the quantitative evaluation of the skin affected by diabetes. In this study, the chronic diabetic skin showed inferior biomechanical skin properties compared to the non-diabetic skin. These findings suggest that the predisposition of diabetic patients to skin disorders may be the result of impaired tissue integrity at baseline. However, it was not confirmed whether such skin changes were related to peripheral neuropathies. 


\section{ACKNOWLEDGMENTS}

I would like to offer special thanks to Moon Kyun Cho, Professor, Department of Dermatology, Soonchunhyang University Seoul Hospital for his great comments. This work was supported by the Soonchunhyang University Research Fund.

\section{REFERENCES}

1. Dyck PJ, Albers JW, Andersen H, Arezzo JC, Biessels GJ, Bril V, et al. Diabetic polyneuropathies: update on research definition, diagnostic criteria and estimation of severity. Diabetes Metab Res Rev 2011;27:620-8.

2. Vinik AI, Maser RE, Mitchell BD, Freeman R. Diabetic autonomic neuropathy. Diabetes Care 2003;26:1553-79.

3. Gin H, Baudoin R, Raffaitin CH, Rigalleau V, Gonzalez C. Non-invasive and quantitative assessment of sudomotor function for peripheral diabetic neuropathy evaluation. Diabetes Metab 2011;37:527-32.

4. Dyck PJ, Kratz KM, Karnes JL, Litchy WJ, Klein R, Pach JM, et al. The prevalence by staged severity of various types of diabetic neuropathy, retinopathy, and nephropathy in a population-based cohort: the Rochester Diabetic Neuropathy Study. Neurology 1993;43:817-24.

5. Gerawarapong C. Association of peripheral autonomic neuropathy and sympathetic skin response in the patients with diabetic polyneuropathy: a pilot study in Thailand. J Med Assoc Thai 2015;98:1222-30.

6. Hoitsma E, Reulen JP, de Baets M, Drent M, Spaans F, Faber CG. Small fiber neuropathy: a common and important clinical disorder. J Neurol Sci 2004;227:119-30

7. Nedelec B, Correa JA, Rachelska G, Armour A, LaSalle L. Quantitative measurement of hypertrophic scar: interrater reliability and concurrent validity. J Burn Care Res 2008;29:501-11.

8. Elsner P. Skin elasticity. In: Berardesca E, Elsner P, Wilhelm KP, Maibach HI, editors. Bioengineering of the skin: methods and instrumentation. Boca Raton (FL): CRC Press; 1995. p. 53-64.

9. Yoon HS, Baik SH, Oh CH. Quantitative measurement of desquamation and skin elasticity in diabetic patients. Skin Res Technol 2002;8:250-4.

10. Forst T, Kann P, Pfutzner A, Lobmann R, Schafer H, Beyer J. Association between "diabetic thick skin syndrome" and neurological disorders in di- abetes mellitus. Acta Diabetol 1994;31:73-7.

11. Park JW, Seo CH, Han SH, Lee YG. Sympathetic influence on biomechanical skin properties after spinal cord injury. Spinal Cord 2011;49: 236-43.

12. Hsu WC, Chiu YH, Chen WH, Chiu HC, Liou HH, Chen TH. Simplified electrodiagnostic criteria of diabetic polyneuropathy in field study (KCIS No. 14). Neuroepidemiology 2007;28:50-5.

13. Kucera P, Goldenberg Z, Kurca E. Sympathetic skin response: review of the method and its clinical use. Bratisl Lek Listy 2004;105:108-16.

14. Smalls LK, Randall Wickett R, Visscher MO. Effect of dermal thickness, tissue composition, and body site on skin biomechanical properties. Skin Res Technol 2006;12:43-9.

15. Dobrev H. In vivo study of skin mechanical properties in psoriasis vulgaris. Acta Derm Venereol 2000;80:263-6.

16. Wohlrab J, Wohlrab D, Meiss F. Skin diseases in diabetes mellitus. J Dtsch Dermatol Ges 2007;5:37-53.

17. Huntley AC. Cutaneous manifestations of diabetes mellitus. Dermatol Clin 1989;7:531-46

18. Sarabu R, Grimsby J. Targeting glucokinase activation for the treatment of type 2 diabetes: a status review. Curr Opin Drug Discov Devel 2005;8: 631-7.

19. Wautier JL, Guillausseau PJ. Advanced glycation end products, their receptors and diabetic angiopathy. Diabetes Metab 2001;27(5 Pt 1):535-42.

20. Reihsner R, Melling M, Pfeiler W, Menzel EJ. Alterations of biochemical and two-dimensional biomechanical properties of human skin in diabetes mellitus as compared to effects of in vitro non-enzymatic glycation. Clin Biomech (Bristol, Avon) 2000;15:379-86.

21. Yosipovitch G, Hodak E, Vardi P, Shraga I, Karp M, Sprecher E, et al. The prevalence of cutaneous manifestations in IDDM patients and their association with diabetes risk factors and microvascular complications. Diabetes Care 1998;21:506-9.

22. Huntley AC. The cutaneous manifestations of diabetes mellitus. J Am Acad Dermatol 1982;7:427-55.

23. Enomoto DN, Mekkes JR, Bossuyt PM, Hoekzema R, Bos JD. Quantification of cutaneous sclerosis with a skin elasticity meter in patients with generalized scleroderma. J Am Acad Dermatol 1996;35(3 Pt 1):381-7.

24. Ryu HS, Joo YH, Kim SO, Park KC, Youn SW. Influence of age and regional differences on skin elasticity as measured by the Cutometer. Skin Res Technol 2008;14:354-8. 